\title{
DETERMINANTES DEL ÉXITO DE LAS PYMES EN LA REPÚBLICA DOMINICANA
}

\section{Determinants of Success of PYMES in Dominican Republic}

\section{J aime Aristy Escuder}

Resumen: Este [artículo] presenta los principales resultados de la encuesta aplicada a 254 Pymes dominicanas con el objetivo de caracterizarlas e identificar sus factores de éxito. El estudio incluye un modelo econométrico que cuantifica la probabilidad de éxito en función de un conjunto de variables que pueden transformase en políticas públicas.

Palabras claves: Pequeñas y medianas empresas, influencia, evolución económica, ingreso promedio, inserción en el mercado, expansión sostenida, éxito, Pymes.

Abstract: In this paper we identify the main determinants, conditioned by legal and institutional environment, of the success of Small and Medium Sized Enterprises (SME) in the Dominican Republic. An econometric model is estimated to quantify the significance of each factor on the probability of success.

Keywords: Small and medium enterprises, influence, economic developments, average income, market insertion, sustained expansion, success, Pymes. 


\section{Introducción}

Las pequeñas y medianas empresas en la República Dominicana, al igual que en el resto del mundo, tienen una influencia determinante sobre la evolución económica. La expansión de estas es indispensable para el crecimiento del ingreso promedio de la población y, en consecuencia, para reducir la pobreza. De esa importancia se desprende que sea fundamental identificar cuáles son los principales factores que explican su inserción en el mercado y expansión sostenida.

El objetivo de esta investigación es cuantificar la relación existente entre el éxito de las Pymes y las variables que ejercen una influencia directa sobre el mismo. Para realizar el estudio se utilizará una encuesta que se llevó a cabo a 254 pequeñas y medianas empresas localizadas en el Distrito Nacional y en Santiago. El cuestionario abarca las características de la empresa; las características del propietario; el enfoque empresarial; y la percepción de los empresarios con relación al apoyo gubernamental.

Una vez obtenida esa información se procede a su análisis descriptivo y posteriormente a la elaboración de un modelo econométrico que permita cuantificar la probabilidad de éxito de las Pymes en el país.

\section{Revisión bibliográfica}

Existe una amplia literatura internacional que estudia los determinantes del desempeño de las pequeñas y medianas empresas.

Spillan y Parnell (2006) realizan un estudio (153 casos) en los Estados Unidos (Maryland, New York y Pennsylvania) que relacionan los recursos de mercadeo orientados hacia el consumidor y el desempeño de las Pymes. Los resultados sugieren que la filosofía de orientación hacia el cliente y una estructura coordinada dentro de la empresa que le de apoyo a esa estrategia de mercadeo orientado hacia el consumidor son los factores que explican un desempeño empresarial superior. 
Al-Mahrouq (2010) realiza un estudio (163 casos) que identifica los principales factores que explican el éxito de las Pymes en Jordania. Los resultados revelan que los factores son: tecnología; estructura de la empresa; estructura financiera; productividad y mercadeo; y recursos humanos.

Lin (1998) presenta los resultados de una investigación (43 casos) sobre los determinantes del éxito de las Pymes en Taiwán. Ella encuentra que las actitudes, la calificación, la orientación hacia el mercado, los métodos operativos son más importantes que los equipos. Asimismo, que la orientación hacia el consumidor y el conocimiento de gestión del fundador son clave en la explicación del éxito de esas empresas. En resumen, los temas relacionados con las personas son más importantes que la estructura y la tecnología.

Raduan Che et al. (2006) presenta los resultados de un estudio (100 casos) donde se identifican los factores de éxito de las Pymes en Malasia. Ella evalúa la relación del desempeño con la iniciativa personal, el capital humano, el conocimiento en la gestión y los programas de apoyo gubernamental. Los empresarios con una elevada dosis de iniciativa personal tienden a sobreponerse ante las restricciones que encuentra. En el estudio se afirma que la educación del empresario, la experiencia laboral y si los padres del empresario tenían una empresa esto estará en estrecha relación con su éxito. Por último, se afirma que el estudio no encontró una relación importante entre los programas de apoyo gubernamental y el éxito de las Pymes.

En el caso de Europa, se aprobó la ley de apoyo a la Pymes, la cual tiene como meta promover su desarrollo y capacidad de generación de empleo en toda la Unión Europea. La ley establece diez principios que sirven de guía al diseño y aplicación de las políticas de apoyo a las Pymes. Entre estas resalta la creación de medidas que hagan que las administraciones respondan oportunamente a las necesidades de esas empresas, minimizando las trabas burocráticas y permitiendo que puedan participar en los concursos que suplen bienes y servicios 
a los gobiernos. Asimismo, se promueve la capacitación gerencial y laboral, y la innovación como mecanismo que favorezca la creación de riqueza y capacidad de crecimiento sostenido.

\section{Descripción de las Pymes dominicanas}

En la presente investigación se definirán las pequeñas y medianas empresas por la cantidad de empleados. Las pequeñas empresas son aquellas que emplean entre 11 y 50 personas. Las medianas empresas emplean entre 51 y 150 personas.

El estudio se fundamenta en una encuesta a 254 empresas realizada en junio de 2010 en Santo Domingo y Santiago. El cuestionario abarca las características de la empresa; las características del propietario y el enfoque empresarial. La persona que respondió el cuestionario fue el propietario de la empresa (37\%), el gerente/ administrador $(20 \%)$ o el encargado del departamento de contabilidad (9\%). En esa sección se presentarán los principales resultados de la encuesta.

\section{Aspectos generales}

En esta sección se presenta una descripción de las pequeñas y medianas empresas (Pymes) basada en una encuesta realizada dentro del marco de la presente consultoría. El 73.2\% de las empresas encuestadas son pequeñas y el $26.7 \%$ son medianas. Las empresas encuestadas representan los sectores manufactura, comercio y servicios. La proporción de cada uno de estos sectores se muestra en la siguiente tabla.

\begin{tabular}{r|ccc}
$\begin{array}{r}\text { Actividad } \\
\text { de la empresa }\end{array}$ & Freq. & Porcentaje & Cum. \\
\hline Manufactura & 90 & 35.43 & 35.43 \\
Comercio & 89 & 35.04 & 70.47 \\
Servicios & 75 & 29.53 & 100.00 \\
\hline Total & 254 & 100.00 &
\end{tabular}


Las Pymes dominicanas se encuentran constituidas formalmente en su mayoría como persona jurídica. Si se realiza una tabulación cruzada con la clasificación de las Pymes por su tamaño se observa que las medianas empresas tienen una mayor probabilidad de estar constituidas como personas jurídicas.

\begin{tabular}{r|cc|c}
$\begin{array}{r}\text { Esta empresa está } \\
\text { constituida formalmente }\end{array}$ & \multicolumn{2}{|c|}{ Tamaño empresa } & \multirow{2}{*}{ Pequeña } \\
Mediana & Total \\
\hline Sí, como persona física & 20.97 & 8.82 & 17.72 \\
Sí, como persona jurídica & 77.96 & 91.18 & 81.50 \\
No & 1.08 & 0.00 & 0.79 \\
\hline Total & 100.00 & 100.00 & 100.00
\end{tabular}

Por lo general, las pequeñas y medias empresas fueron creadas por los propietarios actuales o por algún familiar. Las medianas empresas tienen una mayor probabilidad de ser empresas creadas por algún familiar. Esto puede estar sugiriendo que esas empresas de mayor tamaño fueron creadas por algún familiar, quizás miembro de generaciones anteriores y que han crecido de tamaño.

\begin{tabular}{r|cc|c}
$\begin{array}{r}\text { El propietario tiene esta } \\
\text { empresa porque fue creada }\end{array}$ & \multicolumn{2}{|c|}{ Tamaño empresa } & \multirow{2}{*}{ Pequeña } \\
\hline Por un familiar & 13.51 & 23.88 & 16.27 \\
Por él mismo & 72.97 & 67.16 & 71.43 \\
La compró & 12.43 & 5.97 & 10.71 \\
Por varios socios & 1.08 & 2.99 & 1.59 \\
\hline Total & 100.00 & 100.00 & 100.00
\end{tabular}

La distribución de la fecha de inicio de las operaciones revela que en gran parte comenzaron a operar a partir de finales de los años sesenta. La aplicación de un modelo de desarrollo basado en incentivos 
tributarios debe explicar la creación de empresas desde esa fecha. Se observa que la empresa mediana tiene en promedio mayor tiempo de operación que las pequeñas (i.e., 23 vs. 18 años). El hecho de que tenga 5 años más de operación puede ser un factor que explique la transición de una empresa pequeña hacia una mediana. Esa hipótesis habría que verificarla con un modelo econométrico.

El propietario o un familiar del mismo es quien administra el negocio. El 78.4\% de los encuestados afirma que quien administra el negocio es el dueño o algún familiar.

El número de personas que comenzó a trabajar desde el principio revela que las pequeñas y medianas dominicanas comienzan sus operaciones en su gran mayoría con menos de 30 empleados. La media es de 14 empleados y cuando se descompone por tamaño de empresa se observa que la pequeña inicia operaciones con $10 \mathrm{em}$ pleados y la mediana con 26 personas. Esto sugiere que la empresa mediana es mayor que la pequeña desde el inicio de sus operaciones.

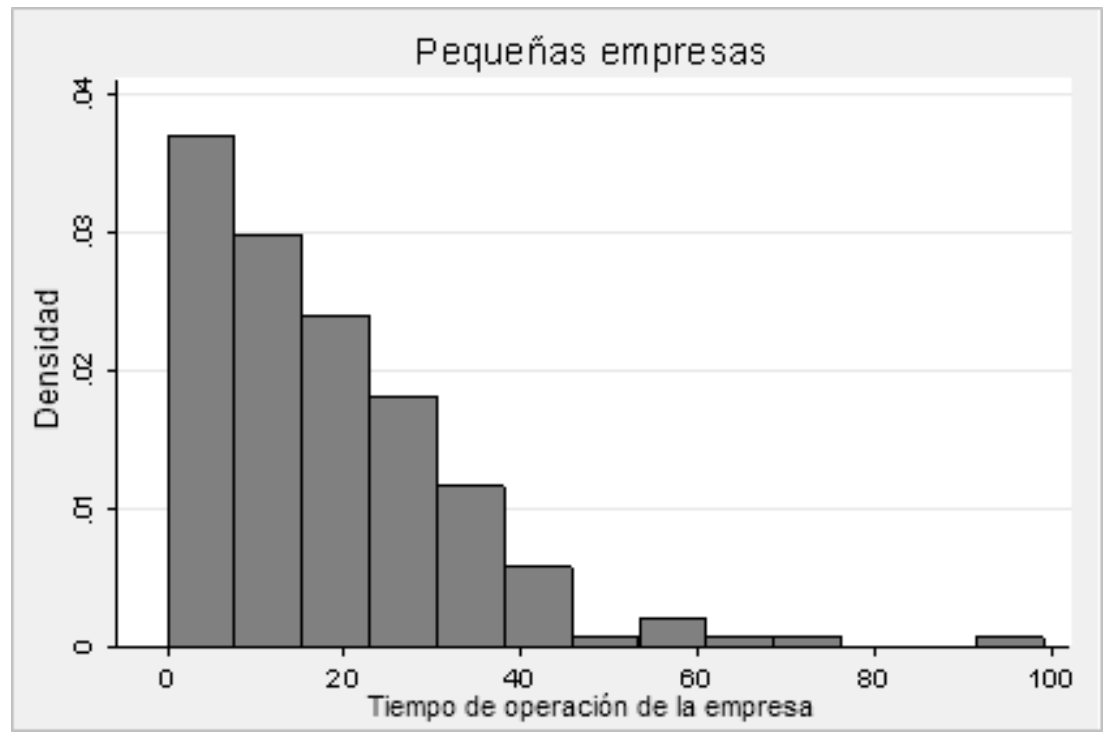




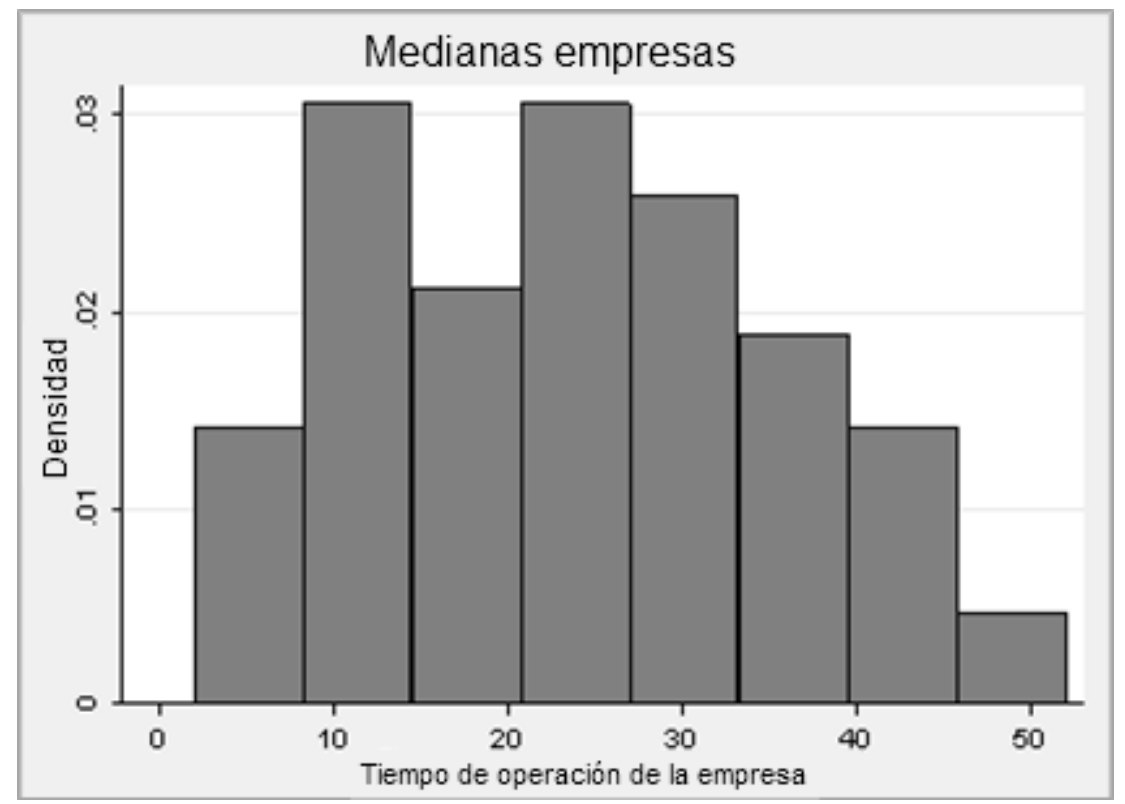

El número de personas que, en promedio, estaba trabajando en las Pymes dominicana en abril de 2010 es de 38 personas, con un mínimo de 11 y un máximo de 150. El promedio del empleo de las pequeñas empresas es de 21 personas y el de la mediana empresa es de 83 trabajadores. Esto sugiere que la mediana empresa ha sido capaz de crear más empleo -en promedio- que las pequeñas desde su inicio de operaciones. Para verificar si las empresas medianas han sido capaces de crear más empleo por año se separó la muestra por tamaño de empresa. Se observa que las medianas crearon en promedio 4 puestos de trabajo por año, mientras que las pequeñas solo crearon 1. La encuesta arroja que el sector manufactura fue el que, en promedio, creo más puestos de trabajo (2.4), seguidas por las actividades del sector comercio (1.8). Al descomponer por tamaño de la empresa, las medianas empresas del sector servicio son las que mayor cantidad de empleos crearon como promedio anual (4.2). Las pequeñas empresas manufactureras son las que más puestos laborales crearon desde el inicio de operaciones (1.5) como promedio anual. 
La principal fuente de recursos para iniciar las Pymes es el ahorro propio o familiar (63.7\%). Los préstamos bancarios o de instituciones de micro-crédito representan el 23\% de la fuente de recursos para dar inicio a la operación empresarial. La pequeña empresa tiende a iniciar en una mayor proporción con el ahorro propio $(66.7 \%$ vs $55 \%)$. Esto sugiere que ambos tipos de empresas no contó inicialmente con un soporte del sistema financiero.

\begin{tabular}{r|cc|c}
$\begin{array}{r}\text { Principal fuente de } \\
\text { dinero para iniciar }\end{array}$ & \multicolumn{2}{|c|}{ Tamaño empresa } & \multirow{2}{*}{ Pequeña } \\
Mediana & Total \\
\hline Ahorros propios & 66.67 & 55.17 & 63.68 \\
Préstamo de familiar & 8.48 & 15.52 & 10.31 \\
Préstamos bancarios & 22.42 & 24.14 & 22.87 \\
Préstamo de suplidores & 1.82 & 1.72 & 1.79 \\
Otros & 0.61 & 3.45 & 1.35 \\
\hline Total & 100.00 & 100.00 & 100.00
\end{tabular}

Los principales clientes de las Pymes son los consumidores finales. Esto se desprende del hecho de que el 43.7\% afirma que los principales clientes son los individuos del barrio o de otros barrios. El segundo tiempo de cliente en importancia son las empresas que los consumen como bien o servicio final (35\%). Solo el $18.5 \%$ de los clientes son empresas que demandan para elaborar otros productos o para la reventa. Las empresas de manufactura suplen en mayor parte a otras empresas, ya sea como consumidor final $(48.9 \%) \mathrm{o}$ como consumidor intermedio (30.0\%). Por su parte las actividades de servicios obtienen el $62.7 \%$ de sus ingresos de los consumidores finales y el $25.3 \%$ de empresas como consumidores finales. El sector comercio suple el $52.8 \%$ de sus bienes a consumidores finales y un $29.2 \%$ a otras empresas.

Las Pymes dominicanas no son exportadoras. Solo el $12.2 \%$ de las empresas encuestadas afirma que exporta algún producto o servicio 
al extranjero. Por sector económico se observa que las actividades con mayor capacidad exportadora, aunque sigue siendo muy baja, es la de manufactura (18.9\%).

Las Pymes encuestadas en su gran mayoría (85\%) utilizan computadoras para registrar sus ventas y llevar la contabilidad de la empresa.

\section{CARACTERÍSTICAS DEL EMPRESARIO}

El propietario de las Pymes es en su mayoría masculino. La mujer solo representa el 10.2\%, mientras que la propiedad conjunta alcanza un $9.5 \%$ de representación. En términos del tamaño de la empresa se observa que la participación masculina aumenta con el tamaño de la empresa, disminuyendo el de la mujer. Cabe resaltar que en el caso de las microempresas, la mujer propietaria representa un $47.6 \% .^{1}$

\begin{tabular}{r|cc|c} 
Género del & \multicolumn{2}{|c|}{ Tamaño empresa } & \multirow{2}{*}{ Total } \\
propietario & Pequeña & Mediana & Ton \\
\hline Hombre(s) & 79.57 & 82.35 & 80.31 \\
Mujer(es) & 11.29 & 7.35 & 10.24 \\
Propiedad conjunta & 9.14 & 10.29 & 9.45 \\
\hline Total & 100.00 & 100.00 & 100.00
\end{tabular}

La edad media del propietario de las Pymes es de 54 años, con un mínimo de 24 y un máximo de 86 años. Solo un $25 \%$ de los propietarios tiene menos de 45 años. La mujer es ligeramente más joven que el hombre propietario de las Pymes. La edad media del hombre es de 54.3 años y la de la mujer es 52.8 años. Se observa que el hombre tiende a permanecer en la empresa por más tiempo.

${ }^{1}$ Véase Guzmán y Ortiz (2007: 27). 


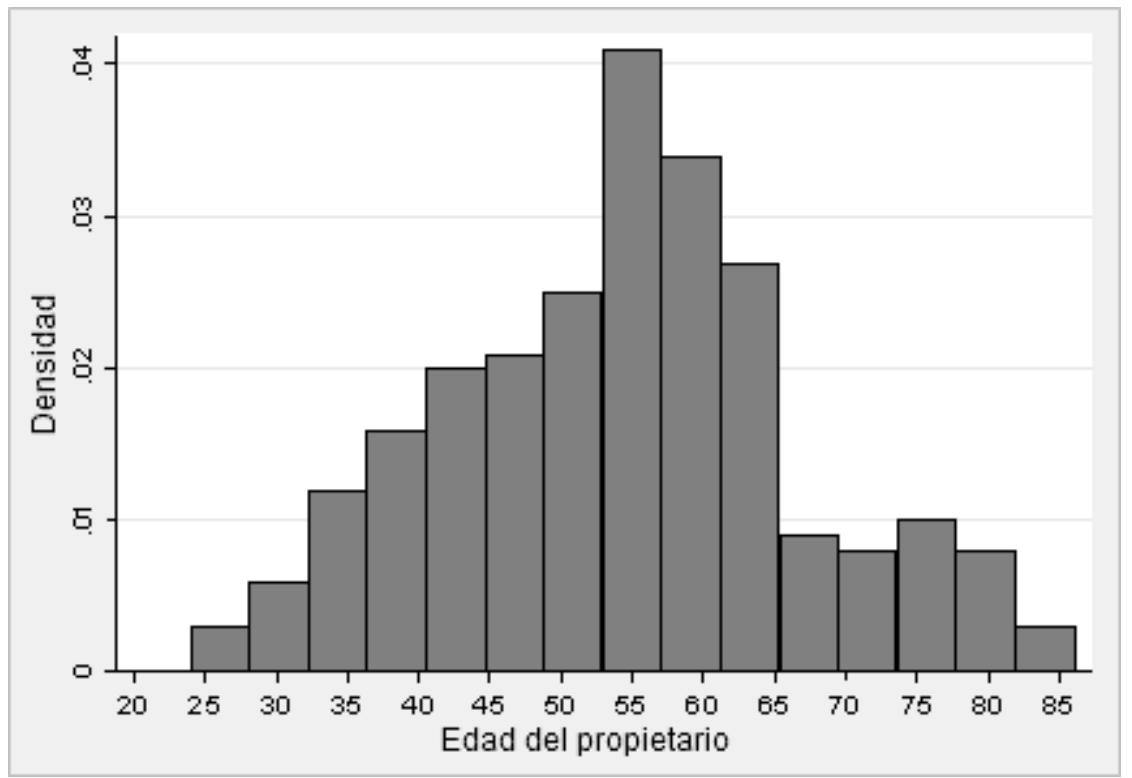

El propietario de las Pymes en el país tiene una calificación elevada para la sociedad dominicana. El 76.9\% de los propietarios posee educación universitaria, de los cuales casi un 13 puntos porcentuales posee un posgrado. Esa realidad contrasta considerablemente con la educación de los propietarios de microempresas. En términos sectoriales se observa que el sector servicios es el que tiene mayor presencia de universitarios $(89.3 \%)$. Por otra parte, las actividades manufactureras son las que tienen el mayor porcentaje de propietarios con educación igual o menor a la primaria $(11.4 \%)$.

La experiencia de los actuales propietarios de Pymes se obtuvo preponderantemente trabajando en otras empresas (46.2\%). El 25.8\% trabajaba en una micro o pequeña empresa y el $20.4 \%$ trabajaba en una empresa grande. No obstante, cabe resaltar que el $36.4 \%$ de las personas encuestadas afirmó que el propietario de esa empresa tenía o tiene un negocio propio $(36.4 \%)$. Solo el $4.9 \%$ de los propietarios actuales de las Pymes estudiaba, lo cual revela algo interesante. La probabilidad de pasar de ser estudiante a poseer una Pyme es casi nula. Eso es compatible con una edad promedio del propietario 
mucho mayor que la edad en que se sale de la universidad. El propietario de las Pymes se inicia en ese tipo de negocios a la edad promedio de 28 años. La trayectoria es: estudiante, empleado y, posteriormente, propietario.

El propietario de la mediana empresa tiene mayores conocimientos de las técnicas de administración (60.3\% vs. 48.9\%), finanzas $(60.3 \%$ vs. $46.2 \%)$, mercadeo (50\% vs. $43.4 \%$ ) que el propietario de la pequeña empresa. El conocimiento en las áreas de recursos humanos $(50 \%$ vs. $47.8 \%$ ) y de la parte operativa $(64.6 \%$ vs. 61.5\%) de la empresa es muy similar entre los propietarios de pequeñas y medianas empresas.

Con esas respuestas se construyó una variable de escala que recogiera el conocimiento del propietario en las técnicas generales de administración de la empresa. Esa variable de conocimiento engloba lo que sabe de administración, finanzas, mercadeo, recursos humanos y la parte operativa del negocio.

La variable conocimiento se construyó mediante el uso de la metodología de la alpha de Cronbach. ${ }^{2}$ Esta permite cuantificar el nivel de correlación entre la escala de medida para la magnitud de la variable conocimiento y las variables que se observaron en la encuesta. El coeficiente de Cronbach es de un $89.4 \%$, lo cual refleja una elevada correlación entre la escala construida y otras posibles escalas que se puedan construir con 5 ítems que traten de medir el conocimiento empresarial de los propietarios de las Pymes.

La siguiente tabla muestra la correlación individual entre los diferentes ítem que conforman a la variable conocimiento. Obsérvese que (exceptuando al ítem e17e, que es la respuesta al conocimiento en la parte operativa del negocio) todas las correlaciones de los ítems individuales con el resto de los ítems (item-rest correlation) son

\footnotetext{
${ }^{2}$ Véase Cronbach, L.J. (1951). Esta metodología permite agregar en una sola variable las influencias de diversas variables que representen el mismo concepto. Esto reduce la presencia de errores de medidas y de multicolinealidad en los modelos econométricos.
} 
superiores al 70\%. Además, la última columna, que mide cuál sería el valor del alpha si se eliminase el ítem correspondiente, arroja que (exceptuando al ítem e17e) todos tendrían un valor inferior al alpha promedio (89.4). Eso justifica la permanencia de esos ítems en la variable "conocimiento" construida con esta metodología.

\begin{tabular}{|c|c|c|c|c|c|c|}
\hline Item & Obs & Sign & $\begin{array}{l}\text { Item-Test } \\
\text { Correlation }\end{array}$ & $\begin{array}{l}\text { Item-Rest } \\
\text { Correlation }\end{array}$ & $\begin{array}{c}\text { Average } \\
\text { Inter-Item } \\
\text { Covariance }\end{array}$ & Alpha \\
\hline $\mathrm{e} 17 \mathrm{a}$ & 247 & + & 0.8989 & 0.8302 & .5119729 & 0.8496 \\
\hline $\mathrm{e} 17 \mathrm{~b}$ & 247 & + & 0.8618 & 0.7763 & .544784 & 0.8626 \\
\hline e17c & 247 & + & 0.8557 & 0.7645 & .543802 & 0.8651 \\
\hline $\mathrm{e} 17 \mathrm{~d}$ & 247 & + & 0.8413 & 0.7441 & .5554623 & 0.8698 \\
\hline e17e & 247 & + & 0.7266 & 0.5869 & .6340531 & 0.9026 \\
\hline Test scale & & & & & .5580149 & 0.8939 \\
\hline
\end{tabular}

La variable conocimiento tiene un rango que va de un mínimo de 1.6 a un máximo de 5. La media de la distribución es 4.25 y la mediana es 4.4, lo cual revela que la masa de la distribución se concentra principalmente hacia los valores mayores. La construcción de esa escala de conocimiento permite concluir que en promedio los propietarios de la mediana empresa tienen ligeramente un mayor conocimiento en administración del negocio que los propietarios de la pequeña empresa. Los resultados revelan que la media del conocimiento de estos últimos es de 4.21 y la media de los propietarios de las medianas empresas es de 4.36 .

En la siguiente tabla se presenta la variable conocimiento excluyendo la pregunta e17e. Nótese que el alpha subió ligeramente.

Test Scale $=$ mean (Unstandardized Items) 
Determinantes del éxito de las Pymes en la República Dominicana

\begin{tabular}{|c|c|c|c|c|c|c|}
\hline Item & Obs & Sign & $\begin{array}{l}\text { Item-Test } \\
\text { Correlation }\end{array}$ & $\begin{array}{l}\text { Item-Rest } \\
\text { Correlation }\end{array}$ & $\begin{array}{c}\text { Average } \\
\text { Inter-Item } \\
\text { Covariance }\end{array}$ & Alpha \\
\hline $\mathrm{e} 17 \mathrm{a}$ & 250 & + & 0.9189 & 0.8478 & .6243159 & 0.8607 \\
\hline $\mathrm{e} 17 \mathrm{~b}$ & 250 & + & 0.9030 & 0.8242 & .6564177 & 0.8698 \\
\hline e17c & 250 & + & 0.8874 & 0.7951 & .6669183 & 0.8800 \\
\hline e17d & 250 & + & 0.8316 & 0.7046 & .732091 & 0.9112 \\
\hline Test scale & & & & & 6699357 & 0.9081 \\
\hline
\end{tabular}

\section{Hipótesis}

Las principales hipótesis a ser evaluadas son las siguientes:

1. La forma de financiamiento del arranque de la empresa influye sobre el éxito de las Pymes.

2. Una estrategia orientada hacia el mercado eleva el desempeño de las Pymes.

3. Una política de capacitación laboral influye positivamente.

4. El acceso al capital mejora el desempeño de las Pymes.

5. La capacidad gerencial del fundador o propietario es determinante del éxito.

6. La estabilidad macroeconómica explica la probabilidad de éxito de las pequeñas y medianas empresas.

7. El apoyo gubernamental directo ${ }^{3}$ (ej., acceso al financiamiento, capacitación, información y acceso a mercados) es un determinante del éxito empresarial.

\footnotetext{
${ }^{3}$ Ese apoyo gubernamental directo puede ampliarse con elementos que definen el marco legal e institucional. Por ejemplo, protección a la propiedad, el estímulo a la innovación, la lucha contra la corrupción y la promoción de la competencia. Véase Cooter y Schafer (2012).
} 
8. Variables demográficas (ej.: edad, género) influyen sobre el desempeño de la empresa.

\section{Ventajas competitivas y obstáculos}

\section{CAPACIDAD GERENCIAL}

En la sección III se observó que los propietarios de la pequeña y la mediana empresa poseen un elevado nivel educativo y un amplio conocimiento en técnicas de administración. Ese acervo de preparación académica y de experiencia contrasta considerablemente con el nivel de calificación de los propietarios de microempresas. De ahí que los propietarios de Pymes tengan más capacidad de hacer crecer sus negocios, basándose en un proceso que permite incrementar las ventas y optimizar su flujo de efectivo, para tener el efectivo necesario para realizar las compras de materias primas y equipos.

\section{ACCESO AL CAPITAL}

El inicio de las Pymes se fundamentó en el uso de recursos propios y prestados. Existe un reconocimiento de la importancia de la acumulación de capital para poder invertir y crecer. La mayoría de los entrevistados muestra estar de acuerdo o muy de acuerdo con la afirmación de que pueden obtener préstamos bancarios y líneas de crédito fácilmente. Debe resaltarse que la mediana empresa tiene mayor facilitad de obtener ese financiamiento. ${ }^{4}$

\footnotetext{
${ }^{4}$ En Guzmán y Ortiz (2007: 41), se muestra en base a una encuesta a Pymes del año 2000, que los obstáculos financieros son percibidos entre los principales limitantes para el desarrollo de esas empresas, principalmente para las más pequeñas. No obstante, en ese estudio solo el 6\% de las Pymes declaró que tienen inconvenientes para acceder al crédito. Esto puede estar sugiriendo que el limitante puede ser el nivel de las tasas de interés, que tradicionalmente ha sido muy elevado en la República Dominicana. En Ortiz y Mena (2007) se afirma que las microempresas tienen menor acceso al financiamiento.
}

510 Ciencia y Sociedad 2012; 37(4): 497-528 
Determinantes del éxito de las Pymes en la República Dominicana

\begin{tabular}{|c|c|c|c|}
\hline \multirow{2}{*}{$\begin{array}{r}\text { Podemos conseguir } \\
\text { préstamos bancarios } \\
\text { y líneas de créditos }\end{array}$} & \multicolumn{2}{|c|}{ Tamaño empresa } & \multirow[b]{2}{*}{ Total } \\
\hline & Pequeña & Mediana & \\
\hline Muy en desacuerdo & 1.63 & 0.00 & 1.20 \\
\hline En desacuerdo & 4.89 & 0.00 & 3.59 \\
\hline Neutral & 9.24 & 4.48 & 7.97 \\
\hline De acuerdo & 48.91 & 50.75 & 49.40 \\
\hline Muy de acuerdo & 35.33 & 44.78 & 37.85 \\
\hline Total & 100.00 & 100.00 & 100.00 \\
\hline
\end{tabular}

\section{ESTABILIDAD Y CAPACITACIÓN LABORAL}

La mano de obra es relativamente estable y entrenable. Casi el 86\% de los encuestados afirma estar en desacuerdo y muy en desacuerdo con la afirmación que establece que los empleados cambian con frecuencia. Esa estabilidad laboral es muy similar entre las pequeñas $(86 \%)$ y las medianas empresas $(85.3 \%)$. Esa característica de la mano de obra permitiría un proceso de aprendizaje que se traduzca en un incremento de la productividad laboral. Cabría preguntarse si esa estabilidad se debe a la existencia de reglamentaciones en el Código Laboral que encarecen el despido de personal, por lo cual los empresarios prefieren retener a los trabajadores (hasta que renuncien) y estos permanecer en el trabajo (hasta que los despidan).

\begin{tabular}{r|cc|c}
\multirow{2}{*}{$\begin{array}{c}\text { En esta empresa los } \\
\text { empleados cambian } \\
\text { con frecuencia }\end{array}$} & \multicolumn{2}{|c}{ Tamaño empresa } & \\
& Pequeña & Mediana & Total \\
\hline Muy en desacuerdo & 35.48 & 47.06 & 38.58 \\
En desacuerdo & 50.54 & 38.24 & 47.24 \\
Neutral & 7.53 & 8.82 & 7.87 \\
De acuerdo & 5.38 & 5.88 & 5.51 \\
Muy de acuerdo & 1.08 & 0.00 & 0.79 \\
\hline Total & 100.00 & 100.00 & 100.00
\end{tabular}


Los beneficios de una mano de obra "relativamente estable" se complementan favorablemente con el hecho de que en la empresa se ofrece o facilita la capacitación. El 79.5\% de los encuestados señaló estar de acuerdo o muy de acuerdo con esa afirmación. El porcentaje es mayor en las medianas empresas $(86.8 \%)$ versus el que se registra en la pequeña empresa $(76.9 \%)$.

\section{ORIENTACIÓN HACIA EL MERCADO ${ }^{5}$}

Las Pymes están orientadas preponderantemente hacia el mercado. Se toman decisiones que tienen como meta mejorar el conocimiento de las necesidades de los clientes y de cómo satisfacerla de la mejor manera posible. Esas empresas interactúan con los clientes, mediante reuniones periódicas para determinar cuáles productos y servicios necesitarán los consumidores en el futuro.

No obstante, la mayoría reconoce que no se realizan encuestas -por lo menos anualmente- para medir la calidad de los productos. Esto significa un bajo nivel de análisis técnico de las relaciones de las empresas con los clientes para determinar la percepción de la calidad de los productos.

Los entrevistados afirman que la capacidad de reacción de la gerencia a los gustos de los clientes es adecuada. La mayoría está en desacuerdo con la afirmación que establece que en la empresa son lentos para determinar los cambios de gusto de los clientes. Además las respuestas permiten señalar que los empresarios se sienten con la información necesaria sobre los problemas que afectan a su negocio.

La capacidad de reacción ante las acciones de la competencia es rápida. La mayoría de los entrevistados mostró estar de acuerdo o muy de acuerdo con la existencia de una reacción rápida cuando la competencia cambia los precios de productos similares. Los empresarios de medianas empresas están más de acuerdo con la rapidez de reacción

\footnotetext{
${ }^{5}$ Esta subsección se basa en la metodología presentada en Spillan y Parnell (2006).
} 
a los movimientos de los precios de la competencia que aquellos que administran pequeñas empresas $(94.1 \%$ vs. $84.9 \%)$.

Incluso los empresarios llegan a afirmar que en sus empresas hay personas que se dedican a planear respuestas a los cambios que se producen en ese tipo de negocios, ya sea en las regulaciones, tecnología o deseos de los clientes. Hay una afirmación más favorable en las empresas de mayor tamaño.

La empresa ejecuta continuamente acciones orientadas hacia el cliente. La mayoría de los entrevistados afirma estar de acuerdo o muy de acuerdo con la sentencia que establece que una vez cada tres meses se realizan reuniones para analizar lo que quieren los empresarios. Se observa que las empresas conocen a sus clientes y tienen datos sobre ellos. Esos datos sobre los clientes se hacen circular en toda la empresa.

El grado de coordinación entre los empleados y departamentos de las empresas es bueno. Los encuestados afirman estar en desacuerdo con la afirmación que establece que existe poca comunicación entre los empleados sobre las necesidades de los clientes. Las respuestas también permiten rechazar la afirmación de que en las Pymes hay poca coordinación entre los diferentes puestos de trabajo. Otro soporte que valida la existencia de coordinación dentro de la empresa es el hecho de que se está de acuerdo con la afirmación que establece que si un cliente pide una modificación de un producto, todos trabajan en conjunto para lograr la satisfacción del consumidor.

La encuesta revela la existencia de una clara orientación hacia el cliente. La mayoría de los encuestados afirma estar de acuerdo o muy de acuerdo con la afirmación que establece que el cliente siempre tiene la razón. Debe señalarse que los propietarios de las pequeñas empresas están más de acuerdo con esa afirmación que los de las medianas empresas. En las Pymes se toma en consideración lo que quiere el cliente y se actúa rápidamente para mejorar ante la insatisfacción de los clientes. Las necesidades de los clientes 
condicionan las actuaciones de las Pymes. "Todo lo que se hace en la empresa es por nuestros clientes": es una afirmación compartida por la mayoría de los encuestados. Sin embargo, se observa que un porcentaje de los encuestados piensa que los clientes se quejan demasiado por cualquier cosa.

Ante las acciones de la competencia para captar clientes, las Pymes reaccionan y buscan la manera de que se queden con ellos. No hay diferencia significativa entre las pequeñas y las medianas empresas. Se debe señalar que en otros estudios la competencia es uno de los "obstáculos" percibidos por las Pymes. ${ }^{6}$

Para recoger la influencia de todas las variables anteriores en una sola que refleje la orientación hacia el mercado de las Pymes se utilizó la metodología de Cronbach y se creó una variable de escala. Los resultados, después de sacar algunas variables con efecto negativo o no significativo, son los siguientes.

\begin{tabular}{c|cccccc} 
& & & & \multicolumn{3}{c}{$\begin{array}{c}\text { Average } \\
\text { Inter-Item }\end{array}$} \\
Item & Obs & Sign & $\begin{array}{c}\text { Item-Test } \\
\text { Correlation }\end{array}$ & $\begin{array}{c}\text { Item-Rest } \\
\text { Correlation }\end{array}$ & $\begin{array}{c}\text { Covariance } \\
\text { Alpha }\end{array}$ \\
\hline e20a & 252 & + & 0.6608 & 0.5486 & .2178155 & 0.7662 \\
e20b & 252 & + & 0.5614 & 0.4518 & .2360115 & 0.7776 \\
e20 & 252 & + & 0.7012 & 0.6110 & .2176732 & 0.7608 \\
e20i & 252 & + & 0.6206 & 0.4766 & 2176311 & 0.7755 \\
e20j & 252 & + & 0.6685 & 0.5295 & .2084445 & 0.7688 \\
e201 & 252 & + & 0.5475 & 0.3902 & .2291701 & 0.7865 \\
e20o & 252 & + & 0.5156 & 0.3896 & .2392631 & 0.7837 \\
e20p & 252 & + & 0.4669 & 0.3388 & .2457138 & 0.7885 \\
e20r & 252 & + & 0.5637 & 0.4784 & .2426499 & 0.7776 \\
e20s & 252 & + & 0.5238 & 0.4278 & .2446584 & 0.7809 \\
e20u & 252 & + & 0.4902 & 0.3815 & .2459889 & 0.7843 \\
\hline Test scale & & & & & .2313654 & 0.7936 \\
\hline
\end{tabular}

${ }^{6}$ Véase Guzmán y Ortiz (2007), p. 41.

514 Ciencia y Sociedad 2012; 37(4): 497-528 


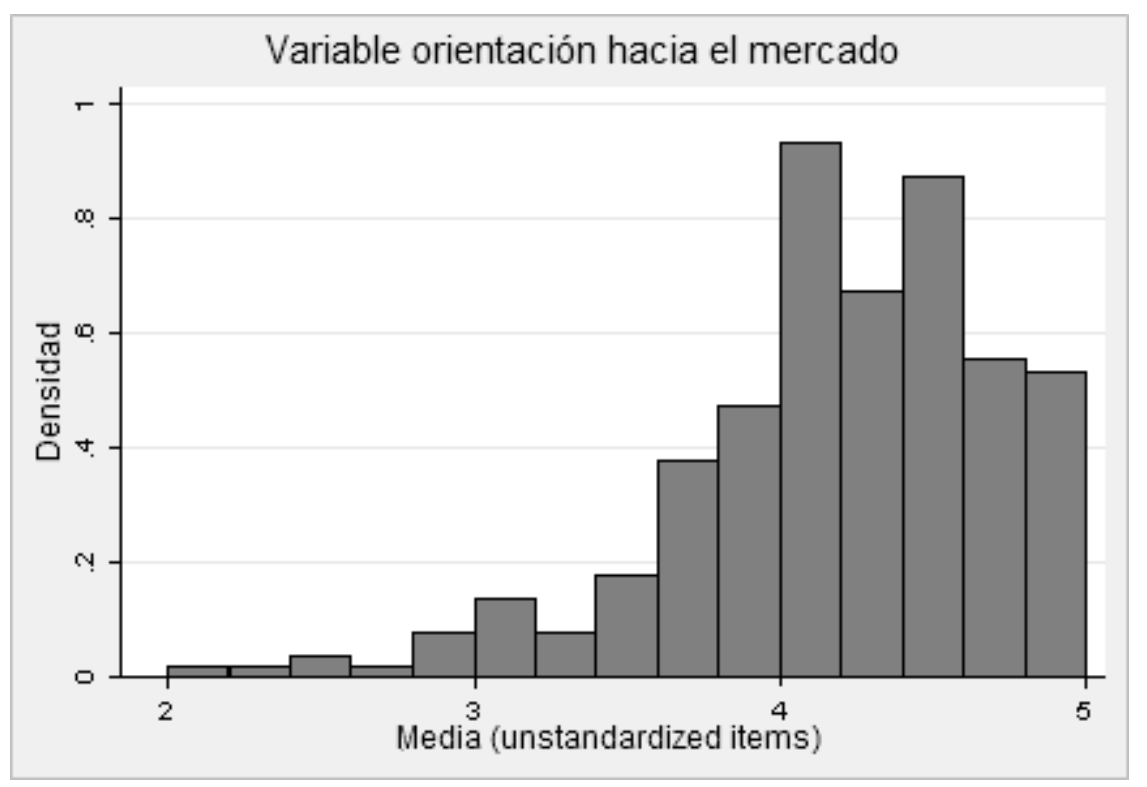

\section{ACCESO A SISTEMAS DE CÓMPUTOS MODERNOS}

Los propietarios de las Pymes afirman que su sistema de cómputos es moderno. Los resultados mostrados en la siguiente tabla revelan que la mediana empresa tiene una mayor probabilidad de poseer un sistema de cómputos moderno.

\begin{tabular}{r|cc|c}
$\begin{array}{r}\text { El sistema de cómputos } \\
\text { puede ser considerado } \\
\text { como moderno }\end{array}$ & \multicolumn{2}{|c}{ Tamaño empresa } & \\
Pequeña & Mediana & Total \\
\hline Muy en desacuerdo & 4.37 & 0.00 & 3.19 \\
En desacuerdo & 13.11 & 2.94 & 10.36 \\
Neutral & 13.11 & 10.29 & 12.35 \\
De acuerdo & 35.52 & 50.00 & 39.44 \\
Muy de acuerdo & 33.88 & 36.76 & 34.66 \\
\hline Total & 100.00 & 100.00 & 100.00
\end{tabular}




\section{APOYO GUBERNAMENTAL DIRECTO}

En el año 2008 se aprobó la Ley 488-08 de incentivo a las micro, pequeñas y medianas empresas de la República Dominicana. El gobierno dominicano ha creado Promipyme con el objetivo de facilitar el acceso al mercado financiero. Asimismo, existe Fomipyme que promueve el desarrollo de garantías para facilitar el acceso al crédito. Lamentablemente, a abril 19 de 2012 no se había aprobado el reglamento que haría operativa la ley 488-08, y el desempeño de Promipyme y Fomipyme ha sido muy limitado. El retraso en la aprobación de ese reglamento es un reflejo de la debilidad institucional que prevalece en la República Dominicana. Debilidad que se combina con trabas burocráticas, ineficiencias en servicios públicos (educación, salud, seguridad, agua, electricidad, entre otros) y corrupción para limitar la capacidad de crecimiento sostenido de los sectores productivos nacionales, en particular de las Pymes.

Los empresarios encuestados afirman en su mayoría que conocen algún tipo de apoyo gubernamental para las Pymes, en especial los que operan las medianas empresas. Sin embargo, la existencia de esa ayuda no se ha traducido en beneficios reales.

La ayuda gubernamental en asistencia técnica es muy limitada. Solo el $11.6 \%$ de los que conocen los programas de apoyo gubernamental afirma que ha logrado conseguir asistencia técnica para su empresa. La casi totalidad de los encuestados afirma que no ha recibido apoyo gubernamental para adquirir equipos y maquinarias. La existencia de ayuda gubernamental tampoco le ha permitido a las Pymes adquirir materias primas o insumos.

El acceso al capital no ha sido facilitado por la intervención del gobierno. Casi el 90\% afirma que a pesar de conocer la existencia de algún tipo de ayuda gubernamental, no ha podido obtener créditos. Esto revela que el radio de acción de Promipyme, como fuente de financiamiento para esas empresas, sigue siendo muy limitado. 
PROMIPYME - Facilita el acceso al mercado financiero.

- Realiza estudios para determinar demanda y necesidades de las Pymes.

- Promueve planes integrales para las Mipymes provisionales.

- Impulsa asociaciones que eleven la competitividad.

- Destinar el 5\% de sus fondos a consultoría y capacitación.

- Garantizar servicio financiero a las Mipymes con el 30\% de los recursos.

- Da preferencia a las Mipymes en las compras estatales.

- Incorporación de los trabajadores a la Seguridad Social.

- Programa para el desarrollo de exportadores en colaboración con el CEI-RD.

- Promover políticas para que las Mipymes protejan el medio ambiente.

- Establecer junto a Preindustria parques industriales, centros tecnológicos, centros de investigación, incubadoras de empresas y centros de desarrollos productivos.

- Promover la formación empresarial junto al INFOTEP.

- Establecer un programa permanente de Desarrollo e Innovación Tecnológica.

FOMIPYME - Destinar el 90\% de sus recursos a actividades crediticias de $1^{\circ}$ y $2^{\circ}$ piso en beneficio de las Mipymes.

- Promover el establecimiento del Sistema de Garantías Recíprocas y Fondos de Garantía para facilitar el acceso al crédito y financiar proyectos. 
A pesar de la existencia del CEI-RD, organismo promotor de la inversión extranjera y de las exportaciones nacionales, la mayoría de los encuestados afirma que no han recibido información sobre los mercados en el extranjero. El gobierno tampoco ha podido ayudar a que la empresa se integre a una cadena productiva o cluster, en especial la pequeña empresa. Las de mayor tamaño han tenido un mayor chance de poder aprovechar ese tipo de apoyo gubernamental.

Con relación a la capacitación, se puede dividir entre el entrenamiento a nivel de gerencia y a nivel de trabajadores. Los resultados revelan que no ha habido un proceso de capacitación a nivel de gerencia. En la capacitación a los trabajadores se nota la mayor presencia de la ayuda gubernamental, aun cuando la mayoría sigue afirmando que el gobierno no ayuda a capacitar a los trabajadores. El mejor aporte al entrenamiento de trabajadores puede ser el resultado de las acciones del INFOTEP que brindan acceso a una amplia variedad de cursos a los empleados de las empresas. Cabe resaltar que la mediana empresa aprovecha más ese tipo de ayuda del gobierno.

\begin{tabular}{|c|c|c|c|}
\hline \multirow{2}{*}{$\begin{array}{r}\text { Capacitar a los } \\
\text { trabajadores }\end{array}$} & \multicolumn{2}{|c|}{ Tamaño empresa } & \multirow[b]{2}{*}{ Total } \\
\hline & Pequeña & Mediana & \\
\hline Sí & 25.51 & 35.00 & 28.26 \\
\hline No & 74.49 & 65.00 & 71.74 \\
\hline Total & 100.00 & 100.00 & 100.00 \\
\hline
\end{tabular}

Para recoger en una sola variable la influencia del gobierno en el éxito de las Pymes se construyó una variable de escala que agrupa la influencia de todas las variables anteriores. Para ello se usó la metodología del alpha de Cronbach.

Test scale $=$ mean (unstandardized items) 
Determinantes del éxito de las Pymes en la República Dominicana

\begin{tabular}{c|cccccc} 
& & & & \multicolumn{3}{c}{$\begin{array}{c}\text { Average } \\
\text { Inter-Item }\end{array}$} \\
Item & Obs & Sign & $\begin{array}{c}\text { Correlation } \\
\text { Coviriance }\end{array}$ & Alpha \\
\hline e22a & 137 & + & 0.6281 & 0.5202 & .0333732 & 0.7571 \\
e22b & 137 & + & 0.7619 & 0.5981 & .0257417 & 0.7422 \\
e22c & 137 & + & 0.5638 & 0.4193 & .0338077 & 0.7692 \\
e22d & 137 & + & 0.7191 & 0.6026 & .0297926 & 0.7398 \\
e22e & 137 & + & 0.7037 & 0.5667 & .0294041 & 0.7447 \\
e22f & 137 & + & 0.4448 & 0.3087 & .0368746 & 0.7832 \\
e22g & 137 & + & 0.6157 & 0.4373 & .0313848 & 0.7698 \\
e22h & 137 & + & 0.6230 & 0.5277 & .0341757 & 0.7589 \\
\hline Test Scale & & & & & .0318193 & 0.7826 \\
\hline
\end{tabular}

\section{COSTO DE LOS SERVICIOS DE ELECTRICIDAD}

La República Dominicana tiene una de las tarifas de electricidad más elevadas de toda América Latina, debido a la histórica participación del Estado Dominicano en ese sector. Esto reduce la competitividad y la rentabilidad de los negocios. Casi la totalidad de los encuestados afirman que el costo de la electricidad en la actualidad es mucho mayor que el costo hace dos años. Esto sugiere la necesidad de tomar medidas que reduzcan el precio de generación para apoyar el desempeño exitoso de las Pymes.

\section{IMPUESTOS}

Los propietarios de las Pymes afirman en su gran mayoría que ahora están pagando mucho más impuestos que hace dos años. La aplicación del Número de Comprobante Fiscal incrementó considerablemente la carga tributaria de esas empresas, reduciéndole su flujo libre de efectivo. Esto es considerado como una limitación al crecimiento y éxito de las Pymes. 


\begin{tabular}{r|cc|c} 
& \multicolumn{2}{|c|}{} & \\
Los impuestos & \multicolumn{2}{|c}{ Tamaño empresa } & \\
para el negocio & Pequeña & Mediana & Total \\
\hline Mucho menor & 1.68 & 0.00 & 1.22 \\
Menor & 1.68 & 3.03 & 2.04 \\
Igual & 8.94 & 10.61 & 9.39 \\
Mayor & 47.49 & 42.42 & 46.12 \\
Mucho mayor & 40.22 & 43.94 & 41.22 \\
\hline Total & 100.00 & 100.00 & 100.00
\end{tabular}

\section{IMPACTO DE LA CRISIS ECONÓMICA Y FINANCIERA MUNDIAL}

La mayoría de los entrevistados afirma que la crisis económica y financiera mundial les afectó negativamente sus empresas. En la siguiente tabla se observa que el $89.6 \%$ de los entrevistados de la mediana empresa afirman que la crisis mundial 2008-2010 les afectó. Asimismo, el 85\% de los representantes de la pequeña empresa bajo análisis también afirma que esa crisis le afectó negativamente. ${ }^{7}$

\section{Factores de éxito}

\section{CREACIÓN DE LA VARIABLE DE ÉXITO}

En el cuestionario se preguntó sobre la situación actual de la empresa con relación a hace dos años. La respuesta se recogió usando la siguiente escala de Likert: mucho menor, menor, igual, mayor y mucho mayor. Las variables relacionadas con éxito son: la rentabilidad de la empresa; las ventas de la empresa; la cantidad de trabajadores en la empresa; participación de la empresa en el mercado; y la inversión con capital propio. En base a esas respuestas se uso la metodología del

\footnotetext{
${ }^{7}$ En Guzmán y Ortiz (2007: 40) se afirma que en la Encuesta de Clima de Inversión realizada por el Banco Mundial en el año 2005, el 60\% de las empresas afirmó que la inestabilidad macroeconómica es un obstáculo a la inversión.
} 
alpha de Cronbach para construir una variable de éxito estandarizada, que posteriormente fue convertida en una variable dicotómica que toma valores de 0 y 1 .

\section{MODELO ECONOMÉTRICO TEÓRICO}

En esa sección se estimará la probabilidad de éxito de las Pymes en base a la información obtenida en la encuesta. Se utilizará un modelo logit binario de la variable exito_d creada de las variables que reflejan un buen desempeño económico de las empresas. El modelo logit binario consiste en la siguiente ecuación:

$$
\operatorname{Pr}\left(\text { exito }_{d}=1 / x\right)=\frac{\exp (\alpha+\beta x)}{1+\exp (\alpha+\beta x)}
$$

\section{ESTIMACIÓN DE LA PROBABILIDAD DE ÉXITO}

A continuación se presentan los resultados de la estimación de 12 modelos econométricos. En estos se evalúa el impacto de la procedencia de los recursos para iniciar el negocio; la edad del propietario; el entorno macroeconómico; la política de orientación hacia el mercado; el apoyo gubernamental; el nivel de capacitación a los empleados; el acceso a préstamos; conocimientos administrativos; frecuencia de cambio del personal; disponibilidad de sistema de cómputos moderno; disponibilidad de efectivo para pagar a los suplidores; disponibilidad de efectivo para el pago de impuestos; género; y el grado de experiencia del propietario previo a tener el negocio actual.

En la siguiente tabla se muestra que la edad es una variable que influye negativamente sobre la probabilidad de éxito. A mayor edad menor probabilidad de éxito de la empresa.

El uso de ahorros propios para iniciar el negocio incrementa la probabilidad de éxito. Esto sugiere que el uso de préstamos desde el inicio puede traducirse en presiones al negocio que desemboquen en su desaparición o empeoramiento del desempeño. No obstante, 
los modelos también reflejan (a un nivel de significancia de un 10\%) que el acceso a préstamos tiene un efecto positivo sobre el desempeño de las empresas. Estos resultados sugieren que existe una clara diferencia entre usar préstamos para arrancar el negocio y usarlos para expandirlo.

La orientación hacia el mercado influye positivamente sobre el éxito de las Pymes. Una estrategia basada en satisfacer al cliente y preparar a la empresa internamente para que cumpla con esa meta se traduce en una mayor probabilidad de éxito.

\begin{tabular}{r|cccc}
\hline Variable & logit1 & logit2 & logit3 & logit4 \\
\hline Ahorro & $0.739^{*}$ & 0.670 & 0.696 & $0.762^{*}$ \\
Edad & $-0.058^{* * *}$ & $-0.058^{* * *}$ & $-0.056^{* * *}$ & $-0.058^{* * *}$ \\
Crisis & $-1.949^{* * *}$ & $-1.919^{* * *}$ & $-1.922^{* * *}$ & $-1.668^{* * *}$ \\
Mercado & $0.770^{* *}$ & 0.310 & & \\
Apoyo & 0.170 & 0.046 & 0.089 & 0.280 \\
Capacitación & & $0.410^{*}$ & $0.520^{* *}$ & \\
Préstamos & & 0.452 & 0.467 & \\
Conocimiento & & & & 0.365 \\
_cons & 0.932 & -0.566 & 0.131 & $2.357^{*}$ \\
\hline $\mathrm{N}$ & 201 & 198 & 200 & 199 \\
r2_p & 0.166 & 0.194 & 0.189 & 0.148 \\
\hline
\end{tabular}

Legenda: ${ }^{*} \mathrm{p}<0.05 ; * * \mathrm{p}<0.01 ; * * * \mathrm{p}<0.001$

El apoyo gubernamental directo, a pesar de tener el signo correcto, no tiene una influencia estadísticamente significativa sobre la probabilidad de éxito de las empresas.

La capacitación de los empleados es un elemento que explica positivamente el éxito de las pequeñas y medianas empresas. Un mayor nivel de capacitación se traduce en mayor productividad y, en consecuencia, en mayor retorno para el empresario.

La crisis macroeconómica y financiera internacional -y obviamente sus secuelas internas- impacta negativamente sobre el desempeño de 
las Pymes. Un entorno económico incierto, con altas tasas de interés, inestabilidad cambiaria, reducción de la demanda agregada, incrementos de costos y volatilidad en los precios, son elementos que influyen negativamente sobre los resultados económicos de esas empresas. La estabilidad macroeconómica es un apoyo gubernamental indirecto.

\begin{tabular}{r|cccc}
\hline Variable & Logit5 & Logit6 & Logit7 & Logit8 \\
\hline Ahorro & 0.697 & $0.863^{*}$ & 0.646 & 0.622 \\
Edad & $-0.056^{* * *}$ & $-0.055^{* * *}$ & $-0.056^{* * *}$ & $-0.058^{* * *}$ \\
Crisis & $-1.923^{* * *}$ & $-2.020^{* * *}$ & $-1.870^{* * *}$ & $-1.925^{* * *}$ \\
Capacitación & 0.520 & $0.470^{* *}$ & $0.514^{* *}$ & $0.527^{* *}$ \\
Préstamos & 0.466 & 0.383 & 0.411 & 0.438 \\
Frecuencia & -0.008 & & & \\
Apoyo & 0.088 & 0.173 & 0.104 & 0.101 \\
Cómputos & & 0.283 & & \\
Dinero_sup s & & & 0.130 & -0.132 \\
Dinero_imp s & & & -0.123 & 0.683 \\
_cons & 0.152 & -0.474 & 200 & 199 \\
$\mathrm{~N}$ & 200 & 197 & 0.190 & 0.194 \\
\hline r2_p & 0.189 & 0.203 & & \\
\hline
\end{tabular}

Legenda: ${ }^{*} \mathrm{p}<0.05 ; * * \mathrm{p}<0.01 ; * * * \mathrm{p}<0.001$

\begin{tabular}{r|cccc}
\hline Variable & Logit9 & Logit10 & Logit11 & Logit12 \\
\hline Ahorro & 0.673 & 0.671 & $0.835^{*}$ & $0.723^{*}$ \\
Edad & $-0.049^{* *}$ & $-0.050^{* *}$ & $-0.058^{* * *}$ & $-0.056^{* * *}$ \\
Crisis & $-1.921^{* * *}$ & $-1.938^{* * *}$ & $-1.970^{* * *}$ & $-2.058^{* * *}$ \\
Capacitación & $0.530^{* *}$ & $0.533^{* *}$ & & $0.626^{* * *}$ \\
Préstamos & 0.459 & 0.444 & & 0.445 \\
Tiempo & -0.012 & -0.011 & & \\
Apoyo & 0.103 & 0.093 & 0.356 & \\
Mujer & & -0.257 & & \\
Experiencia & & & $0.655^{*}$ & $0.728^{*}$ \\
_cons & -0.009 & 0.122 & $3.254^{* * *}$ & -0.873 \\
N & 198 & 198 & 190 & 187 \\
r2_p & 0.188 & 0.189 & 0.157 & 0.215 \\
\hline
\end{tabular}

Legenda: ${ }^{*} \mathrm{p}<0.05 ;{ }^{* *} \mathrm{p}<0.01 ;{ }^{* * *} \mathrm{p}<0.001$ 
La experiencia previa influye positivamente sobre la probabilidad de éxito de las empresas. Esto confirma las conclusiones de otros estudios que describían el hecho de que los propietarios de pequeñas y medianas empresas pasaban por un proceso de aprendizaje que iba de ser empleado en otra empresa.

Ser mujer no influye estadísticamente (aunque tiene un signo negativo) sobre el desempeño de las Pymes.

\section{Predicción}

Para realizar la predicción de un resultado de éxito se utilizará el modelo 12 cuyos resultados se presentan a continuación. Note que las variables son estadísticamente significativas y tienen los signos esperados.

Iteration 0: $\quad \log$ pseudolikelihood $=-129.61585$

Iteration 1: $\log$ pseudolikelihood $=-103.16121$

Iteration 2: $\quad \log$ pseudolikelihood $=-101.77702$

Iteration 3: $\quad \log$ pseudolikelihood $=-101.7454$

Iteration 4: $\quad \log$ pseudolikelihood $=-10.74537$

Logistic Regression

Number of obs $=187$

Wald chi2(6) $=50.08$

Prob $>$ chi2 $=0.0000$

Log Pseudolikelihood $=-101.74537$

Pseudo R2 $=0.2150$

\begin{tabular}{r|cccccc}
\hline Éxito_d & Coef. & $\begin{array}{c}\text { Robust } \\
\text { Std. Err. }\end{array}$ & $\mathbf{z}$ & $\mathbf{p}>|\mathbf{z}|$ & \multicolumn{2}{c}{$[\mathbf{9 5 \%}$ Conf. Interval] } \\
\hline Ahorro & .7229973 & .3670067 & 1.97 & 0.049 & .0036734 & 1.442321 \\
Edad & -.0564066 & .0143296 & -3.94 & 0.000 & -.0644921 & -.028321 \\
Crisis & -2.058411 & .4497313 & -4.58 & 0.000 & -2.939868 & -1.176953 \\
Experiencia & .7277345 & .2962543 & 2.46 & 0.014 & -.1470866 & 1.308382 \\
Capacitación & .6264017 & .1699509 & 3.69 & 0.000 & .2933041 & .9594993 \\
Préstamos & .4449959 & .2453035 & 1.81 & 0.070 & -.0357901 & .9257819 \\
_cons & -.8734379 & 1.51716 & -0.58 & 0.565 & -3.847018 & 2.100142 \\
\hline
\end{tabular}


Los resultados de la estimación se muestran la tabla siguiente. El valor mínimo es un $2.7 \%$ y el máximo un $97.6 \%$.

\section{Probabilidades de éxito}

\begin{tabular}{ccclr}
\hline & Percentiles & Smallest & & \\
$1 \%$ & .0492412 & .0265162 & & \\
$5 \%$ & .1144073 & .0492412 & & \\
$10 \%$ & .1531701 & .0587032 & Obs & 195 \\
$25 \%$ & .2784239 & .0720902 & Sum of Wgt. & 195 \\
& & & & \\
$50 \%$ & .4986083 & & Mean & .5051422 \\
& & Largest & Std. Dev. & .2572027 \\
$75 \%$ & .6952315 & .9548523 & & \\
$90 \%$ & .8622084 & .9675415 & Variance & .0661532 \\
$95 \%$ & .9278836 & .9707801 & Skewness & .0370563 \\
$99 \%$ & .9707801 & .9764073 & Kurtosis & 1.888183 \\
\hline
\end{tabular}

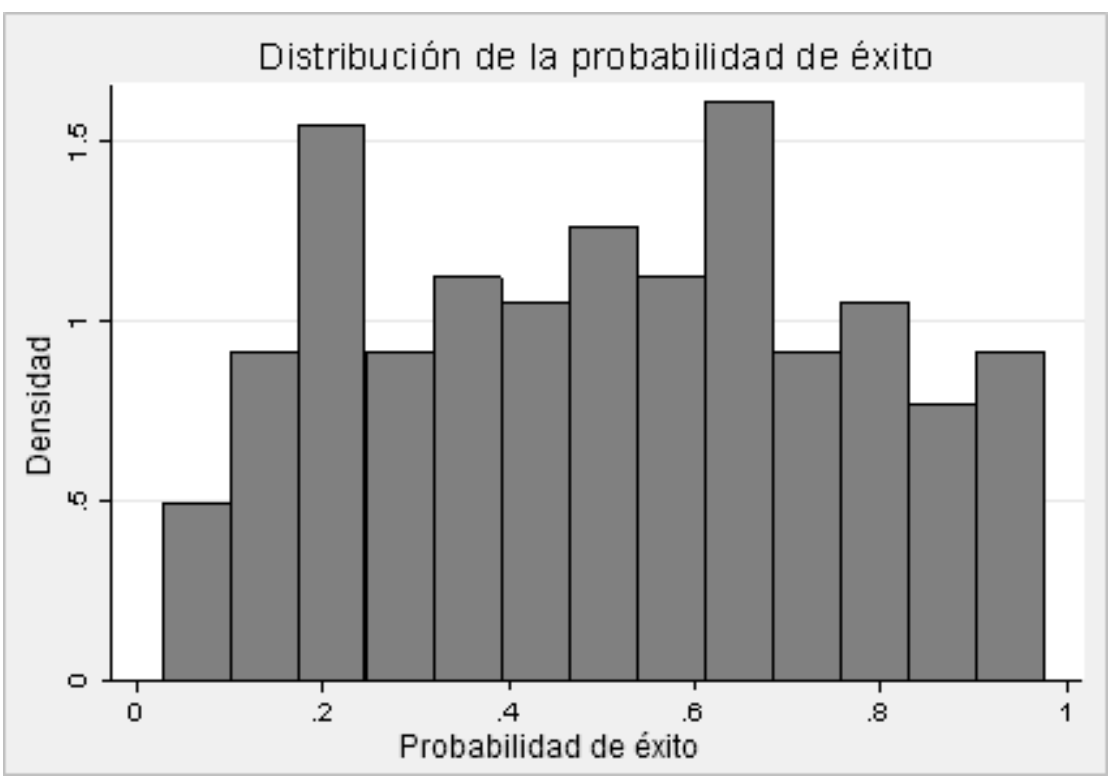




\section{Conclusiones y recomendaciones}

Los resultados preliminares permiten identificar los principales factores que explican el éxito de las Pymes $^{8}$ en la República Dominicana. Entre estos se encuentran: la forma de obtención de los recursos al iniciar el negocio; la edad del empresario; el grado de conocimiento de gestión empresarial; la orientación hacia el mercado y el consumidor; la capacitación de la mano de obra; el acceso a préstamos (después que se inicia la operación); y el entorno macroeconómico. La actual política de apoyo gubernamental directo no ejerce una influencia estadísticamente significativa sobre la probabilidad de éxito de esas empresas.

De esos resultados se pueden elaborar políticas públicas que influyan sobre cada uno de esos determinantes para lograr elevar la probabilidad de éxito de las Pymes. Entre esas políticas se pueden definir aquellas que faciliten capital de riesgo que promueva el arranque sin tener la presión de pagar las cuotas de un préstamo. Asimismo, el aumento del conocimiento de la gestión empresarial sería un apoyo importante para los empresarios, que permitiría crear una empresa orientada hacia el mercado. Programas de capacitación laboral que eleven la productividad y eficiencia de la mano de obra. Y sobre todo, una política macroeconómica que mantenga la estabilidad y que permita la correcta asignación de los recursos productivos.

La aplicación de esas medidas de políticas públicas elevaría el ritmo de crecimiento de las Pymes y mejoraría su aporte al desarrollo nacional.

\footnotetext{
${ }^{8}$ Se entrevistaron más de 50 empresas de manufacturas, más de 40 comercios y más de 45 empresas de servicios para obtener y plantear estos resultados.
} 


\section{Bibliografía}

Al-Mahrouq, M. (2010). "Success Factor of Small and Medium Sized Enterprises (SMEs): The Case of Jordan". Anadolu University Journal of Social Science.

Cooter, R. y Hans-Bernd Schaffer (2012). Solomon's Knot: How Law can End the Poverty of Nations. Princeton University Press.

Cronbach, L.J. (1951) "Coefficient Alpha and the Internal Structure of Test" Psychometrika 16, 3, 297-334.

Guzmán, R. y M. Ortiz (2007) Tamaño, eficiencia y uso de la capacidad instalada en las pequeñas \& medianas empresas de la República Dominicana. FondoMicro y Grupo de Consultoría Pareto.

Raduan Che, R.; Kumar, Naresh and Li Yen, L: (2006). "Entrepreneurs Success Factors and Escalation of Small and Medium Sized Enterprises in Malaysia”. Journal of Social Science.

Spillman, J. y J. Parnell (2006). "Marketing Resources and Firm Performance Among SMEs." European Management Journal Vol. 24, Nos. 2-3, pp. 236-245. Elsevier.

Tambunan, T. (2005). "Promoting Small and Medium Enterprises with a Clustering Approach: A Policy Experience from Indonesia". Journal of Small Business Management.

Van Zyl, C. (2007). "Key Performance Predictors of Small and Micro Tourism Enterprises in South Africa: Philosophical and Methodological Underpinnings". 16 ${ }^{\text {th }}$ EDAMBA Summer Academy.

Wilson, A. "Determinants of Success: Small and Medium Sized Enterprises Selling Goods and Services to the Federal Government". National-Louis University. 
Yeh-Yun Lin, C. (1998). "Success Factor of Small and Medium Sized Enterprises in Taiwan: An Analysis of Cases". Working Paper.

\section{Jaime Aristy Escuder}

Licenciado en Economía Summa Cum Laude INTEC (1985); doctorado en Economía Universidad de Barcelona (1989); magíster en Matemáticas Puras PUCMM (1994); master of Science en Financial Mathematics Universidad de Chicago (2003). Desde 1989 ha sido profesor de Economía Matemática y de Econometría en la PUCMM, INTEC y en la Universidad Católica Santo Domingo, tanto a nivel de maestría como a nivel de licenciatura. Ha escrito y colaborado en 10 libros publicados en la República Dominicana o por casas editoriales extranjeras (Fondo de Cultura Económica y McGraw-Hill). Autor de ensayos que han sido presentados en las Universidades de MIT, Stanford, Harvard y Londres.

Email: jaimearisty@gmail.com

Recibido: $10 / 10 / 2012$ Aprobado: 12/11/2012 